\title{
Trends of tuberculosis treatment outcomes at Mizan-Aman general hospital, southwest Ethiopia: A retrospective study
}

\author{
Wegderese Sintayehu ${ }^{1}$, Abebe Abera ${ }^{2}$, Teklemichael Gebru ${ }^{3}$, Temesgen Fiseha ${ }^{1}$ \\ ${ }^{1}$ Mizan-Aman General Hospital, Mizan, Ethiopia \\ ${ }^{2}$ Department of Nursing, Jimma University, Jimma, Ethiopia \\ ${ }^{3}$ Department of Public Health, Aman Health Science College, Mizan, Ethiopia
}

Email addresses:

teklemichaelgebru@gmail.com (T. Gebru),temafiseha@gmail.com (T. Fiseha)

To cite this article:

Wegderese Sintayehu, Abebe Abera, Teklemichael Gebru, Temesgen Fiseha. Trends of Tuberculosis Treatment Outcomes at Mizan-Aman General Hospital, Southwest Ethiopia: A Retrospective Study. International Journal of Immunology. Vol. 2, No. 2, 2014 , pp. 11-15.

doi: $10.11648 /$ j.iji.20140202.11

\begin{abstract}
Background: TB continues to be a major public health problem in Ethiopia, which ranks seventh by estimated number of cases among the 22 TB high-burden countries. TB Treatment will only be effective if the patient completes the regimen which includes a combination of drugs recommended by the physicians. Incomplete treatment may result in prolonged excretion of bacteria which may lead to increased morbidity and mortality and spread of the disease. Objectives: to assess the trends of tuberculosis treatment outcomes at Mizan Aman General Hospital, Bench Maji Zone, Southwest Ethiopia, from September 2010 to August 2013. Methods: A retrospective study design was conducted at the TB clinic of Mizan general Hospital by analyzing the data of registered tuberculosis patients. The original case records were carefully reviewed, analyzed and interpreted to determine descriptive statistic such as frequencies and proportion of variables. The results of the study were presented using tables and line diagram. The study was approved ethically by Jimma University, college of public health and medical science, department of Nursing. Result: about 2043 TB patient record was retrieved in this study period from 2010- 2013. Of these, $1207(58.00 \%)$ were males and $874(42.00 \%)$ were females. The magnitude of TB case during the study period were smoothly declined in the first four consecutive six months 382, 312, 212 and 116, respectively, whereas in the fifth six month the TB case dramatically increased to 547 however in the last phase reduces to 472. During the study period $362(17.72 \%)$ completed the treatment, $79(3.87 \%)$ cured, $4(0.20 \%)$ defaulted, 1575 $(76.99 \%)$ transferred out to other health facility, $25(1.22 \%)$ were died but no one could failed the treatment regimen. Conclusion: The trends of overall TB diagnosed cases were reduced in the first two years but it increases in the third year. The treatment outcomes; high in transfer out and low in cure rate was observed. Thus, Behavioral change communication education emphasized on early detection and treatment of TB cases is important strategies to reduce TB burden.
\end{abstract}

Keywords: TB, Trend, Treatment Outcome

\section{Introduction}

\subsection{Background}

Tuberculosis (TB), an infectious disease caused by the bacillus Mycobacterium Tuberculosis (MTB), is one of the leading causes of morbidity and mortality world-wide, and ranks as the second leading cause of death from infectious diseases next to the human immunodeficiency virus (HIV) $(1,2)$. Globally in 2012 , an estimated 8.6 million people developed TB and there were 1.3 million deaths from the disease (including an estimated 1.1 million cases and 320 000 deaths among HIV-positive people). About $58 \%$ and $27 \%$ of the 8.6 million people who developed TB in 2012 were in the Asian and African regions respectively $(1,3)$.

TB continues to be a major public health problem in Ethiopia, ranks seventh by estimated number of cases among the 22 TB high-burden countries. In 2012 WHO estimated that there were 230,000 TB cases and 16000 deaths from TB; with an estimated 23,000 cases and nearly 5,600 deaths occurred among HIV-positive people $(1,4)$. The incidence of $\mathrm{TB}$ cases and mortality from $\mathrm{TB}$ are 
unacceptably large given that most are preventable and curable if people can access health care for a diagnosis and the right treatment is provided.

Detection and treatment of new cases in the DOTs strategy, was developed by the WHO for the prevention and control of TB in the early 1990s, is believed to be the most valuable strategy for TB control; and is considered as a cost-effective health intervention for reducing the incidence and death of TB in developing countries. Current antituberculosis therapy consists of a combination of drugs taken over a period of at least 6 months for new patients and 8 months for retreatment patients $(1,5)$.

Although, the key targets of TB control in DOTs were to detect the disease and treat the cases, and to ensure complete their treatment and get cured; delayed presentation for treatment, incomplete treatment or poor compliance as well as treatment interruption or default; a phenomenon that contributes to prolonged infectiousness and increase the risk of drug resistance, relapse, and death, are the major challenge that TB programmes face in resource-constrained settings where the health care services are not well developed (6,7). (8-10).

Hence, assessing trends of TB and its treatment outcomes are important in order to design disease management program in an effort to stop the continued transmission of TB within the community and to reduce drug resistance, relapse or death among affected individuals (11-13). However, despite DOT short course TB treatment is being practiced in Bench Maji Zone, trends of the disease have not been assessed yet in the area. Therefore, the aim of this study was to assess trends and treatment outcomes of TB in Bench Maji Zone, with special emphasize on Mizan hospital, Southern Ethiopia.

\section{Methods}

A retrospective study design was conducted among TB patients registered at Mizan-Aman General Hospital located in Bench Maji Zone Southwest Ethiopia from February 17 to March 14/2014. The hospital serves for the population of Bench Maji and two adjacent zones. The health facilities within these zones register and treat all diagnosed TB patients under the DOTS. All diagnosed TB cases are registered at the unit registers, compiled at the district registers, and reported to the zonal and regional TB programme. Once diagnosed with TB, patients are provided with anti-TB medication and care free of charge. The hospital provides services for approximately 6679 inpatient and 50386 outpatient attendances a year coming to the hospital from the catchment population of about 1.5 million people. Out of the outpatient attendants the hospital has a total of 1165 registered new cases of TB who came to the TB clinic for follow up, and 404 of them are smear-positive pulmonary TB patients. The study was conducted from September 2010 up to August, 2013.

The Source Population of the study was all patient cards/charts of tuberculosis registered. While the Study
Population was all patient cards/charts of tuberculosis registered and put on DOTS in the hospital during the study period from September 2010 to August 2013 were reviewed. But, incomplete patient cards were excluded from the study.

Data was collected by trained nurse professional using structured checklist. The checklist contains patient's demographic characteristics, type of TB (smear-positive PTB, smear- negative PTB, and Extra PTB), immunodeficiency virus (HIV) status, treatment outcomes (cured, completed treatment, defaulted, treatment failure, died or transferred out). Treatment outcomes were evaluated in accordance with the National Tuberculosis and Leprosy Control Program (NTLCP) adopted from the WHO. The data collection process was cheeked on a daily base.

The collected data were entered into a computer using SPSS version 20.0 software. A summary descriptive analysis was made using graphical presentation, calculating summary statistics (frequency and percentages). Outputs were organized and presented in tables, graphs and narratives. Normality and the outliers were checked by pox plot and/or scatter plot.

The study was approved by health research and post graduate coordinating office, college of Public health and medical science of Jimma University. Letter of cooperation was obtained from Bench Maji zone health department and the hospital after informing the purpose of the study.

\section{Result}

\subsection{Patient Characteristics}

Table 1. Patient characteristics of study subjects ( $n=2043)$, Mizan Aman General Hospital, Sep 2010 - Aug 2013.

\begin{tabular}{lll}
\hline Variables and response category & Frequency & Percent (\%) \\
\hline Sex & & \\
Male & 1169 & 57.22 \\
Female & 874 & 42.78 \\
Age group (in years) & & \\
$\leq 14$ & 220 & 10.77 \\
$15-24$ & 498 & 24.38 \\
$25-34$ & 657 & 32.16 \\
$35-44$ & 343 & 16.79 \\
$45-54$ & 204 & 9.99 \\
$55-64$ & 92 & 4.50 \\
$\geq 65$ & 29 & 1.42 \\
TB type & & \\
Smear positive pulmonary TB & 510 & 24.96 \\
Smear negative pulmonary TB & 1043 & 51.05 \\
TB lyphadinophathy & 441 & 21.59 \\
Others & 9 & 0.44 \\
TB category & & \\
Pulmonary TB & 1553 & 76.02 \\
Extra pulmonary TB & 450 & 22.03 \\
HIV status (n = 2016) & & \\
Positive & 328 & \\
Negative & 1650 & \\
\hline
\end{tabular}


A total of $2043 \mathrm{~TB}$ patients were registered at Mizan Aman General Hospital from 2010- 2013. Of these, 1169 (57.2\%) were males and $874(42.78 \%)$ were females. Among the study participants $657(32.16 \%)$ were in the age group from 25 to 34 . Out of the total 2043 cases, 1043 $(51.05 \%)$ were smear negative pulmonary TB cases. In terms of patients category $1,553(76.02 \%)$ were pulmonary TB and 450 (22.03\%) were extra pulmonary TB. About 328 $(16.05 \%)$ of the study participants were HIV positive (Table 1).

\subsection{Treatment Outcomes}

Of the 2043 TB patients who were registered at the hospital during the study period, 347 (16.98\%) were completed the treatment, $181(8.86 \%)$ cured, $4(0.20 \%)$ defaulted, $1486(72.74 \%)$ were transferred out to other health facility, $25(1.22 \%)$ died but no one could failed the treatment regimen. Regarding to smear positive treatment outcome $181(79.39 \%)$ was cure rate, 37(16.23\%) complete and $9(3.95 \%)$ deaths. The treatment success rate (it was computed as: completed the treatment with negative TB bacteria result at the end of treatment (cured) plus completed the treatment but without bacteriology result at the end of treatment) was 218 (95.61\%) (Table 2).
Table 2. TB treatment outcome $(n=2043)$, Mizan Aman General Hospital, Sep 2010 - Aug 2013

\begin{tabular}{lll}
\hline Variables and response category & Frequency & Percent (\%) \\
\hline TB treatment outcome (all type) & & \\
Treatment completed & 347 & 16.98 \\
Cured & 181 & 8.86 \\
Default & 4 & 0.20 \\
Failed & 0 & 0.00 \\
Transfer out & 1486 & 72.74 \\
Death & 25 & 1.22 \\
TB treatment outcome (smear +ve) & & \\
Complete & 37 & 16.23 \\
Cured & 181 & 79.39 \\
Defaulter & 1 & 0.44 \\
Death & 9 & 3.95 \\
TB treatment success rate ( smear + ve) & & \\
Success & 218 & 95.61 \\
Not success & 10 & 4.39 \\
\hline
\end{tabular}

\subsection{Trends of TB Treatment Outcome}

The proportion of TB treatment outcome during our study period were smoothly improved the treatment complete and cure rates however defaulter and death rates were relatively reduced. In the case of transfer out there were dramatic reductions in the second year, but it increased by more than three folds in the last year of study period (Table 3 and Fig. 1).

Table 3. Trends of TB tretment outcomes (n=2043), Mizan Aman general hospital, Sep 2010 - Aug 2013

\begin{tabular}{|c|c|c|c|c|c|c|}
\hline \multirow[b]{2}{*}{ Time in years } & \multicolumn{6}{|c|}{ Treatment outcome No(\%) } \\
\hline & Treatment completed & Cured & Default & Failed & Transfer out & Death \\
\hline 2011 & $95(4.65)$ & $50(2.45)$ & $4(0.20)$ & 0.00 & $538(26.33)$ & $7(0.34)$ \\
\hline 2012 & $101(4.94)$ & $47(2.30)$ & 0.00 & 0.00 & $173(8.47)$ & $14(0.69)$ \\
\hline 2013 & $151(7.39)$ & $84(4.11)$ & 0.00 & 0.00 & $775(37.93)$ & $4(0.20)$ \\
\hline
\end{tabular}

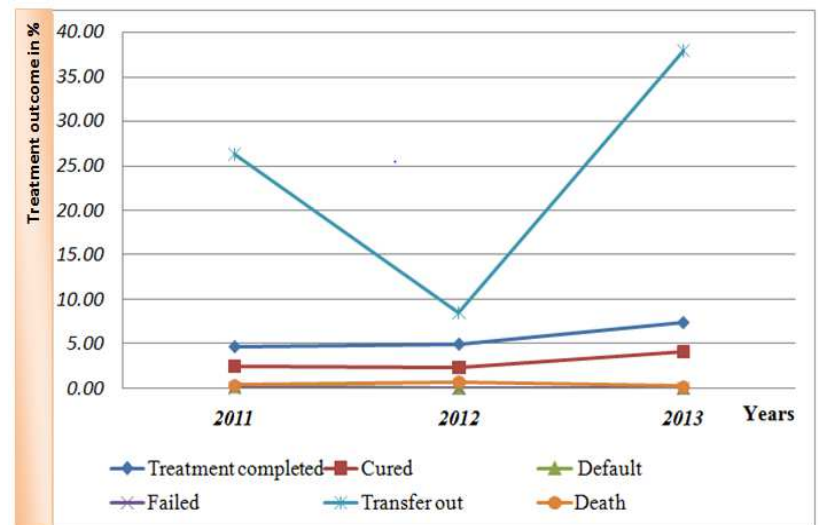

Figure 1. Trends of TB tretment outcomes ( $n=2043)$, Mizan Aman general hospital, Sep 2010 - Aug 2013

\section{Discussion}

In this facility based retrospective study, data on the treatment outcome; demographic characteristics, types of TB and trends across the study period were assessed in Bench Maji zone Mizan Aman general hospital. The high proportion of all forms of TB observed among males compared to females in this study was consistent with study conducted in Gambella regional and Harer Felege Hiwot referral hospitals which reported $54.5 \%$ and $58.46 \%$ male TB cases, respectively $[12,20]$. This might be attributed to either males are more likely to develop the disease or are more likely to utilize health service and diagnose their cases than females.

The overall trend of TB in this study was gradually increasing with age until 34 years $(10.77 \%$ to $32.16 \%)$ and then showed a decline as age increased (32.16\% to $1.42 \%)$. This was consistent with the findings reported in Gambella hospital study $(25.10 \%$ to $28.14 \%$ and $28.14 \%$ to $0.69 \%$, respectively) [12]; that might be as the result of study area location that is near to gold mining, in which youths and early adult age people are prone to come for job causing high proportion of TB cases in these age groups

Majority of patient who were registered during the study period were affected by smear negative pulmonary TB $(51.05 \%)$ and this finding was supported by a Gambella hospital study that reported $50.35 \%$ [12], this might be due to the occurrence of HIV infection. This study also found that the occurrence of pulmonary TB was high as compared to extra pulmonary TB. As to TB HIV co-infection; this study reported $16.05 \%$ TB HIV co-infection which is in agreement with the current Ethiopian Federal Ministry of Health report by $2009 / 10$, reporting $15.00 \%$ TB HIV co-infection [19]. 
The trend of $\mathrm{TB}+$ treatment outcome was found to be improved by cured rate and treatment completeness from first to third years of the study period. On the other hand, the trend of defaulter and death rates were decreased. The overall proportion of cured were $3.87 \%$ which is quite low and this might be due to high transfer out rate $(76.99 \%)$ observed in the hospital. Furthermore, the cure rate of TB smear positive was $62.70 \%$, which was low as compared with the nationally expected level $(85 \%)$ and this difference might be due to poor data registration after follow-up AFB examination at the TB clinic and/or inability to send patients for follow-up AFB examination during scheduled standard treatment period.

The defaulter rate $(0.20 \%)$ revealed in this study was lower than in other studies conducted in the country; $36.4 \%$ and $6 \%$ at Gondar University Teaching Hospital and Southern Ethiopia, respectively [16, 17]. This lower defaulter rate might be due to proper supervision and health education in the study area. The overall proportion of death $(1.22 \%)$ in this study was lower than death rate reported from Addis Ababa (4\%) and the national figure (3\%) [18]. The observable difference might be due to defaulter rates in those previous studies were high and/or lack of follow up and defaulter tracing mechanism. Furthermore, the death rates of $\mathrm{TB}$ smear positive in this study $(7.14 \%)$ were higher than the study conducted in Addis Ababa [19]. This might be due to high prevalence of HIV in the area.

The treatment success rate of TB smear positive found by this study at TB clinic in MIizan Aman General Hospital was satisfactory $(95.61 \%)$. This result is consistent with 2005 WHO report on global tuberculosis control, the treatment success rate among 22-high burden countries varied from $60 \%$ in Uganda to $93 \%$ in China, with an average of $83 \%$ in WHO report, 2005 [5].

\section{Conclusion}

The trend of overall TB cases was reduced in the first two years but it increases in the last year. Furthermore, the overall trends of TB treatment outcomes, particularly treatment complete and cure rate were smoothly increased within the last three years. However, the trend of death rate and defaulters were decreased. On the other hand, the overall trend of transfer out was found as high. Perhaps, the treatment success rate of TB smear positive was found at satisfactory improvements. Cognizant of this fact, we recommend that community mobilization, advocacy, and treatment outcome monitoring program should be design and/or strengthening to reduce the burden of tuberculosis.

\section{Competing Interests}

The authors declare that they have no competing interests.

\section{Authors' Contributions}

WS was involved in the conception, design, analysis, interpretation, report writing and manuscript writing. AA, TG and TF had been involved in the analysis, interpretation, report writing and manuscript writing. All authors read and approved the final manuscript version submitted for publication.

\section{Acknowledgements}

We are highly grateful for all participants of the study, supervisors of data collection and data collectors for their worthy effort and participation in this study. We are also thankful to Mizan-Aman General Hospital for funding this study.

\section{References}

[1] World Health Organization. Global Tuberculosis Report 2013.WHO/HTM/TB/2013.11. Geneva, Switzerland: WHO, 2013. http://whqlibdoc.who.int/hq/2013/ WHO_HTM_TB_2013.11.pdf Accessed November 2013.

[2] Dennis L. Kasper, Eugene Braunwald, Anthony Fauci, Stephen Hauser, Dan Longo, J. Larry Jameson. Harrison's Principles of Internal Medicine. 17th ed. 2008.

[3] HIV-Associated TB Facts 2013 [Internet]. World Health Organization; 2013. Available from: http://www.who.int/tb/challenges/hiv/

[4] WHO World Health Organization Website. Global TB Report 2010. [Internet]. Available from: http://www.who.int/tb/country/data/download/en/index.html.

[5] World Health Organization. Addressing poverty in TB control. WHO report 2005. WHO/HTM/TB/2005.352. Geneva, Switzerland: WHO, 2005. http://whqlibdoc.who.int/hq/2005/ WHO_HTM_TB_2005.352.pdf Accessed November 2013.

[6] Thomas C (2002) A literature review of the problems of delayed presentation for treatment and non-completion of treatment for tuberculosis in less developed countries and ways of addressing these problems using particular implementations of the DOTS strategy. J Manag Med 16: 371-400.

[7] Borgdroff, Floyd K and Broekmans JF. Intervention to reduce tuberculosis mortality and transmission in low- and middle-income countries. Bulletin on the World Health Organization 80, 217-227.

[8] Brudney K \& Dobkin J (1991) Resurgent tuberculosis in New York City: human immunodeficiency virus, homelessness, and the decline of tuberculosis control programs. The American Review of Respiratory Disease 144, 745-749.

[9] Daniel OJ, Oladapo OT \& Alausa OK (2006) Default from tuberculosis treatment programme in Sagamu, Nigeria. Nigerian Journal of Medicine 15, 63-67. 
[10] Sevim T, Atac G, Gungor G et al. (2002) Treatment outcome of relapse and defaulter pulmonary tuberculosis patients. The International Journal of Tuberculosis and Lung Disease $6,320-325$.

[11] Liao et al. Assessing trends and predictors of tuberculosis in Taiwan. BMC Public Health. 2012, 12:29.

[12] Demeke. Trend of Tuberculosis and Treatment Outcomes in Gambella Region with Special Emphasize on Gambella Regional Hospital, Western Ethiopia. J Mycobac Dis. 2013; $3(2)$.

[13] Becerra MC, Freeman J, Bayona J, et al. Using treatment failure under effective directly observed short-course chemotherapy programs to identify patients with multidrugresistant tuberculosis. Int J Tuberc Lung Dis 2000; 4: 10814.

[14] World Health Organization (2009) Global Tuberculosis Control. Addis Ababa, Ethiopia: Country Profile.

[15] Bench Maji Zone 2010 Anual Report. 2011.

[16] Yassin MA, Datiko DG, Tulloch O, Markos P, Aschalew M, et al. Innovative Community-Based Approaches Doubled Tuberculosis Case Notification and Improve Treatment Outcome in Southern Ethiopia. PLoS ONE. 2013, 8(5):

[17] Tessema B, Muche A, Bekele A, Reissig D, Emmrich F, et al. (2009) Treatment outcome of tuberculosis patients at Gondar University Teaching Hospital, Northwest Ethiopia. A fve year retrospective study. BMC Public Health 9: 371

[18] Federal Ministry of Health (FMOH) (2000) Planning and Programming Department. Health and Health Related Indicators. E.C, Addis Ababa, Ethiopia.

[19] Getahun B, Ameni G, Biadgilign S, Medhin G () Mortality and associated risk factors in a cohort of tuberculosis patients treated under DOTS programme in Addis Ababa, Ethiopia, 2011. BMC Infect Dis 11: 127.

[20] Fantahun Biadglegne , Berhanu Anagaw4 , Tewodros Debebe1, Belay Anagaw5 et al

[21] A retrospective study on the outcomes of tuberculosis treatment in Felege Hiwot Referral Hospital, Northwest Ethiopia,2013. International Journal of Medicine and Medical Sciences, 5(2): 85-91, 